\title{
Expression of VEGF and HIF-I $\alpha$ in locally advanced cervical cancer: potential biomarkers for predicting preoperative radiochemotherapy sensitivity and prognosis
}

This article was published in the following Dove Press journal:

OncoTargets and Therapy

23 May 2016

Number of times this article has been viewed

\author{
Pengfeng Zhu' \\ Yangjun $\mathrm{Ou}^{2}$ \\ Yishan Dong' \\ Peizhen $X u^{\prime}$ \\ Lei Yuan ${ }^{3}$ \\ 'Department of Gynecology, \\ 2Department of Pathology, Changzhou \\ Maternity and Children Health \\ Care Hospital Affiliated to Nanjing \\ Medical University, Changzhou, \\ Jiangsu, ${ }^{3}$ Department of Gynecology, \\ Obstetrics and Gynecology Hospital, \\ Fudan University, Shanghai, People's \\ Republic of China
}

\begin{abstract}
Locally advanced cervical cancer (LACC) is an early-stage cervical cancer characterized by a local tumor diameter of $\geq 4 \mathrm{~cm}$. Patients with LACC have a relatively poor prognosis. Although preoperative radiochemotherapy (PRCT) might offer a valuable opportunity for subsequent radical surgery, surgeons should also consider the nonresponsive rate, the adverse effects of PRCT, and the surgical complications before designing a treatment plan. Therefore, biomarkers for predicting PRCT sensitivity and prognosis in patients with LACC are of high importance. We investigated the prognostic significance of vascular endothelial growth factor (VEGF) and hypoxia inducible factor-1 $\alpha$ (HIF-1 $\alpha)$ in patients with LACC. A total of 43 patients with LACC who underwent PRCT (one course each of intravenous chemotherapy and after-loading intracavitary brachytherapy followed by a radical hysterectomy) during the period 2009-2014 were included in this study. VEGF and HIF-1 $\alpha$ expression levels were evaluated by immunohistochemistry in LACC lesions before and after PRCT. In addition, we analyzed the association of these proteins with the clinical response and pathological findings of pelvic lymph node metastasis (PLNM) after the subsequent surgery. The total clinical response rate was $81.39 \%$ after PRCT, including five complete responses and 30 partial responses. VEGF and HIF-1 $\alpha$ expression before PRCT was significantly higher than after PRCT (VEGF: $85.71 \%$ vs $66.67 \%$; HIF- $1 \alpha$ : $83.33 \%$ vs $59.52 \%, P<0.05$ ). In addition, the same trend was found in patients with PLNM compared to those without PLNM (VEGF: $100 \%$ vs $77.78 \%$; HIF-1 $\alpha$ : $100 \%$ vs $74.07 \%, P<0.05)$. The areas under the receiver operating characteristic curves were 0.896 and 0.835 when using pre-PRCT VEGF and HIF-1 $\alpha$ expression levels, respectively, to diagnose PLNM in patients with LACC. Serial detection of VEGF and HIF- $1 \alpha$ demonstrated a sensitivity of $66.67 \%$ and specificity of $88.89 \%$. These findings suggest that VEGF and HIF- $1 \alpha$ expressions are potential biomarkers for PRCT and have great clinical significance for the prediction of PRCT response and prognosis in patients with LACC.
\end{abstract}

Keywords: locally advanced cervical cancer, LACC, preoperative chemoradiotherapy, PRCT, vascular endothelial growth factor, VEGF, hypoxia inducible factor- $1 \alpha, \mathrm{HIF}-1 \alpha$, prognosis

\section{Introduction}

Locally advanced cervical cancer (LACC) is an early-stage cervical cancer that is characterized by a local tumor diameter of $\geq 4 \mathrm{~cm}$. Patients with LACC have a relatively poor prognosis. As recommended by the National Comprehensive Cancer Network (NCCN) guidelines, the treatment modalities for this type of cancer are direct radical hysterectomy, radical surgery following neoadjuvant chemotherapy, and 
radical radiochemotherapy (RCT). However, these treatment modalities have similar clinical effects. ${ }^{1}$ In recent years, preoperative RCT (PRCT) has been gradually used in clinical applications and has attracted extensive attention because it offers the option for surgery and the opportunity to retain ovarian function for young patients. However, varying PRCT responses have been documented, and the response rate is currently reported at $\sim 72 \%-93.7 \%$. $^{2,3}$

According to the International Federation of Gynecology and Obstetrics (FIGO) and NCCN guidelines, pelvic lymph node metastasis (PLNM) is currently not included in the clinical staging of cervical cancer. However, PLNM is closely associated with the development of rational treatment measures and the prognosis of cervical cancer. If PLNM can be accurately predicted before PRCT, it is possible to prevent radical RCT following radical surgery and change to radical chemotherapy instead, which would promptly reduce the suffering of patients by avoiding the complications of RCT and surgery. Therefore, preventing nonresponsiveness to PRCT and predicting the prognosis of patients with LACC have become a hot topic of research. Hypoxia is a common phenomenon that occurs within large LACC lesions. ${ }^{4,5}$ Hypoxic cells are resistant to RCT, and hypoxia inducible factor- $1 \alpha$ (HIF-1 $\alpha$ ) is an important transcription factor that is known to regulate cell biology under specific hypoxic conditions in tumors and is activated in tumor cells. This activation facilitates tumor growth, which in turn induces neovascularization. Moreover, vascular endothelial growth factor (VEGF) is the most critical pro-angiogenic factor and is closely associated with HIF-1 $\alpha$. Generally speaking, hypoxia upregulates VEGF gene expression levels and also increases its transcriptional activity in VEGF-regulating signal transduction pathways. These processes promote neovascularization in cervical cancer and create conditions for continuous development, local invasion, and distant metastasis of cervical cancer cells.

Therefore, we hypothesized that VEGF and HIF-1 $\alpha$ could be potential markers for predicting the PRCT response and prognosis in patients with LACC. In this study, we analyzed the clinical response of patients with LACC to PRCT. We also analyzed the expression of VEGF and HIF-1 $\alpha$ before and after PRCT and assessed their association with PRCT response and PLNM to provide new insights into LACC treatment.

\section{Patients and methods}

\section{Patient selection and clinical treatment regimens}

According to the 2009 FIGO staging system, this study included 43 patients with stage IB2 or IIA2 cervical cancer (maximum cervical tumor size of 4-5 cm) who were admitted to our hospital between January 2009 and December 2014. The diagnosis was confirmed pathologically by cervical biopsy, and the patients did not receive any previous treatment. The age range was 36-68 years, with an average age of $49.88 \pm 9.19$ years. There were 28 cases with stage IB2, 15 cases with stage IIA2, 37 cases with cervical squamous cell carcinoma, and six cases with adenocarcinoma. All patients underwent a pelvic computed tomography scan following hospitalization, and no enlarged lymph node shadows were found. Moreover, the patients had no chemotherapy contraindications, other malignancies, or severe internal diseases. Informed consent was obtained from all patients, and the study was approved by the Ethical Review Committee of Changzhou Maternal and Child Health Care Hospital affiliated to Nanjing Medical University in the People's Republic of China.

Intravenous chemotherapy was first introduced on day 1. The chemotherapy regimen utilized an intravenous infusion of paclitaxel $\left(135 \mathrm{mg} / \mathrm{m}^{2}\right)$ and nedaplatin $\left(80 \mathrm{mg} / \mathrm{m}^{2}\right)$. Three days following chemotherapy, after-loading intracavitary brachytherapy was performed using an ${ }^{192} \mathrm{Ir}$ high-dose-rate after-loading brachytherapy machine at an 8 Gy dose. Clinical evaluations were conducted weekly during the treatment. Patients with no response or disease progression were treated with radical radiotherapy, whereas patients with a response underwent radical hysterectomy 2 weeks post radiotherapy. Both ovaries were retained for patients younger than 45 years old.

Following the preoperative adjuvant therapy, patients underwent weekly gynecological examinations with two deputy chief physicians (or medical staff with higher titles) to perform a clinical evaluation of tumor volume reduction and cervical morphology. Tumor volume was measured via ultrasound examinations. The short-term PRCT response of the solid tumors was evaluated using the World Health Organization evaluation criteria. ${ }^{6}$ The short-term response rate was calculated as follows:

$\underset{\text { response rate }}{\text { Short-term }}=\frac{\text { Complete response }(\mathrm{CR})+\text { Partial response }(\mathrm{PR})}{\text { Total case number }} \times 100 \%$

\section{VEGF and HIF-I $\alpha$ immunohistochemistry}

Prior to PRCT, a colposcopic biopsy of the cervical tumor was performed. A radical hysterectomy was performed 2 weeks post PRCT, and the resected tumor lesions were used in the immunohistochemical assays. Immunohistochemistry 

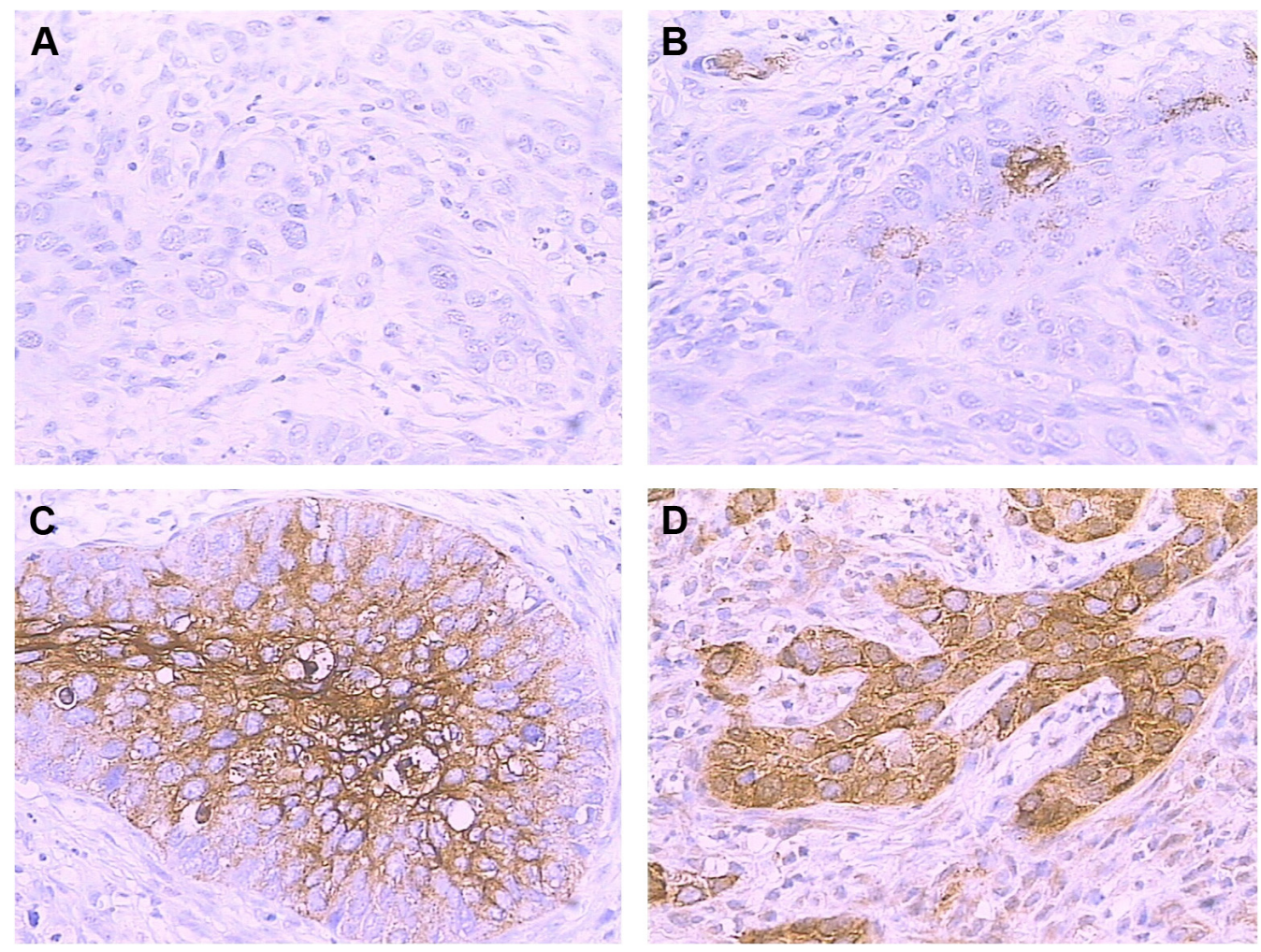

Figure I Immunohistochemical staining of VEGF in LACC lesion.

Notes: Original magnification $\times 400$. (A) Negative, (B) weak expression, (C) moderate expression, (D) strong expression.

Abbreviations: LACC, locally advanced cervical cancer; VEGF, vascular endothelial growth factor.

reagents were all purchased from Maixin Biotech Co., Ltd. (Fuzhou, Fujian, People's Republic of China). The streptavidin-peroxidase-biotin method was utilized in accordance with the manufacturer's instructions. Tissue sections were first incubated with mouse monoclonal anti-VEGF and anti-HIF antibodies at a dilution of 1:50, respectively. Then, a secondary antibody (Supervision_Universal [Anti-Mouse/Rabbit] Detection Reagent) and horseradish peroxidase were applied.

Positive VEGF staining was indicated by the presence of brownish-yellow granules in the cytoplasm, while HIF- $1 \alpha$ staining was mainly present in the cytoplasm with very little expression in the nucleus (Figures 1 and 2). Staining was evaluated with a nomogram as reported by Qiu et al, which incorporates both the proportion of positive staining cells and the staining intensity. ${ }^{7}$ Briefly, the percentage of positive cells was scored as 0 ( $0 \%$ positive cells), 1 (1\%-25\% positive cells), 2 ( $26 \%-50 \%$ positive cells), and $3(>50 \%$ positive cells). The intensity of immunostaining was scored as 0 (negative), 1 (weak), 2 (intermediate), and 3 (strong). The intensity score $(0-3)$ was multiplied by the percentage score (0-3) and a final score was assigned $0-1$ (negative), 2-3 (+, weak expression), 4-5 (++, moderate expression), and $6(+++$, strong expression). The results of the VEGF and HIF- $1 \alpha$ assays were determined by two professional pathologists using a double-blind method. Disagreement was addressed and reviewed by a senior physician.

\section{Statistical analyses}

Continuous data were analyzed using a paired $t$-test and a completely random $t$-test. Categorical data and intergroup rates were analyzed using the chi-square test. A $P$-value $<0.05$ indicated significance. All analyses were performed with SPSS software, Version 20.0 (IBM Corporation, Armonk, NY, USA).

\section{Results}

Following PRCT, five patients had a CR, including four cases of stage IB2 and one case of stage IIA2. Thirty patients had a PR, including 19 cases of stage IB2 and eleven cases of stage IIA2. Seven patients had stable disease, including five cases of stage IB2 and two cases of stage IIA2. One patient had progressive disease, which was diagnosed as stage IIA2 cervical adenocarcinoma. This patient had no tumor volume reduction and exhibited a progressive trend of disease following one course each of chemotherapy and brachytherapy. As a result, this patient was transferred for 

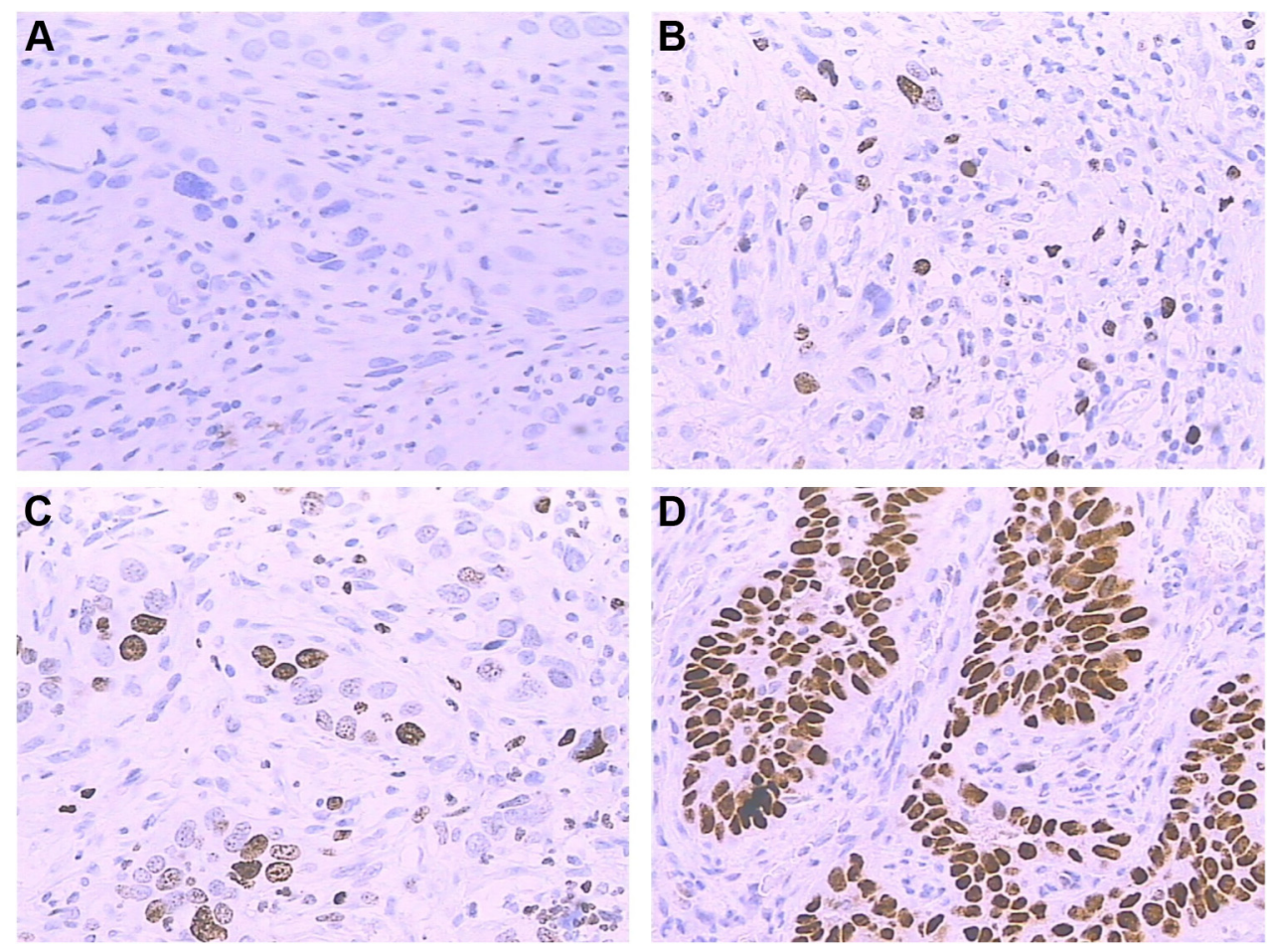

Figure 2 Immunohistochemical staining of HIF-I $\alpha$ in LACC lesion.

Notes: Original magnification $\times 400$. (A) Negative, (B) weak expression, (C) moderate expression, (D) strong expression.

Abbreviations: HIF-I $\alpha$, hypoxia inducible factor-I $\alpha$; LACC, locally advanced cervical cancer.

radical radiotherapy. Therefore, the total response rate was $81.39 \%$ (35/43) with a CR rate of $11.63 \%$ (5/43) and a PR rate of $69.76 \%(30 / 43)$.

The VEGF and HIF- $1 \alpha$ expression rates before and after PRCT are shown in Table 1.The VEGF and HIF-1 $\alpha$ expression rates before PRCT were $85.71 \%$ (36/42) and $83.33 \%$ (35/42), respectively, and the corresponding rates after PRCT were 66.67\% (28/42) and 59.52\% (25/42), respectively. Significant differences were detected when comparing both expression levels before and after PRCT $(P<0.05)$. In addition, the change in both expression levels for the same individual pre and post PRCT was also linked to the clinical responses $(P<0.05)$.

VEGF and HIF- $1 \alpha$ expression rates after PRCT were $0 \%$ $(0 / 5)$ and $0 \%(0 / 5)$ in patients with a CR; 70\% (21/30) and

Table I VEGF and HIF-I $\alpha$ expressions before and after PRCT (n)

\begin{tabular}{lllllll}
\hline Group & $\mathbf{n}$ & VEGF & & & HIF-I $\alpha$ & \\
& & Negative & Positive & & Negative & Positive \\
\hline Before PRCT & 42 & 6 & 36 & 7 & 35 \\
After PRCT & 42 & 14 & 28 & & 17 & 25 \\
$\chi^{2}$ & & 4.20 & & 5.83 & \\
$P$-value & & 0.040 & & 0.016 & \\
\hline
\end{tabular}

Abbreviations: HIF-I $\alpha$, hypoxia inducible factor-I $\alpha$; PRCT, preoperative chemoradiotherapy; VEGF, vascular endothelial growth factor.
$63.33 \%(19 / 30)$ in patients with a PR; and 100\% (7/7) and $85.71 \%(6 / 7)$ in patients with stable disease, respectively (Table 2). The expression rates were significantly different according to the varying clinical responses $(P<0.05)$.

In the non-PLNM group, VEGF and HIF-1 $\alpha$ expression rates before PRCT were 77.78\% (21/27) and 74.07\% (20/27), respectively. In contrast, in the PLNM group, VEGF and HIF-1 $\alpha$ were expressed in all cases. There were significant differences in VEGF and HIF-1 $\alpha$ expression between the nonPLNM and PLNM groups before PRCT $(P<0.05$; Table 3$)$.

Table 2 Association between clinical response to PRCT and expression of VEGF and HIF-I $\alpha$

\begin{tabular}{llll}
\hline Clinical response & $\mathbf{n}$ & $\begin{array}{l}\text { VEGF-positive } \\
\text { cases }(\mathbf{n})\end{array}$ & $\begin{array}{l}\text { HIF-I } \alpha \text {-positive } \\
\text { cases }(\mathbf{n})\end{array}$ \\
\hline CR & 5 & 0 & 0 \\
PR & 30 & 21 & 19 \\
SD & 7 & 7 & 6 \\
PD & 1 & $-^{a}$ & $-^{a}$ \\
$\chi^{2}$ & & 13.65 & 9.53 \\
$P$-value & & 0.001 & 0.009
\end{tabular}

Notes: ${ }^{2}$ The patient with PD after PRCT was changed to radical radiotherapy and was excluded from the following $\mathrm{IHC}$ evaluations.

Abbreviations: $\mathrm{CR}$, complete response; HIF-I $\alpha$, hypoxia inducible factor-I $\alpha$; IHC, immunohistochemistry; PD, progressed disease; PR, partial response; PRCT, preoperative chemoradiotherapy; SD, stable disease; VEGF, vascular endothelial growth factor. 
Table 3 Association between VEGF and HIF-I $\alpha$ protein expression before PRCT and PLNM

\begin{tabular}{|c|c|c|c|c|c|c|c|c|c|}
\hline \multirow[t]{2}{*}{ PLNM } & \multirow[t]{2}{*}{$\mathbf{N}$} & \multicolumn{4}{|c|}{$\begin{array}{l}\text { VEGF expression } \\
\text { before PRCT }\end{array}$} & \multicolumn{4}{|c|}{$\begin{array}{l}\text { HIF-I } \alpha \text { expression } \\
\text { before PRCT }\end{array}$} \\
\hline & & - & + & ++ & +++ & - & + & ++ & +++ \\
\hline Negative & 27 & 6 & 9 & 12 & 0 & 7 & 8 & 9 & 3 \\
\hline Positive & 15 & 0 & 0 & 7 & 8 & 0 & 1 & 5 & 9 \\
\hline$\chi^{2}$ & & 22.74 & & & & 14.33 & & & \\
\hline$P$-value & & 0.001 & & & & 0.002 & & & \\
\hline
\end{tabular}

Notes: -, Negative; +, week expression; ++, moderate expression; +++, strong expression.

Abbreviations: HIF-I $\alpha$, hypoxia inducible factor-I $\alpha$; PLNM, pelvic lymph node metastasis; PRCT, preoperative chemoradiotherapy; VEGF, vascular endothelial growth factor.

Based on the pre-PRCT VEGF and HIF- $1 \alpha$ protein expression scores, we obtained the receiver operating characteristic (ROC) curve of VEGF expression for predicting PLNM in patients with LACC. The area under the ROC curve was $0.896(P=0.001)$, with a $95 \%$ confidence interval of $0.803-0.989$. When predicting PLNM in patients with LACC according to HIF-1 $\alpha$ expression, the area under the ROC curve was $0.835(P=0.002)$, with a $95 \%$ confidence interval of $0.712-0.957$ (Figure 3).

When using the combined VEGF and HIF-1 $\alpha$ protein expression scores, five points were defined as the diagnostic cutoff. A serial diagnosis of PLNM by the combination of VEGF and HIF- $1 \alpha$ protein expression had a sensitivity of $66.67 \%$ and a specificity of $88.89 \%$. The positive predictive

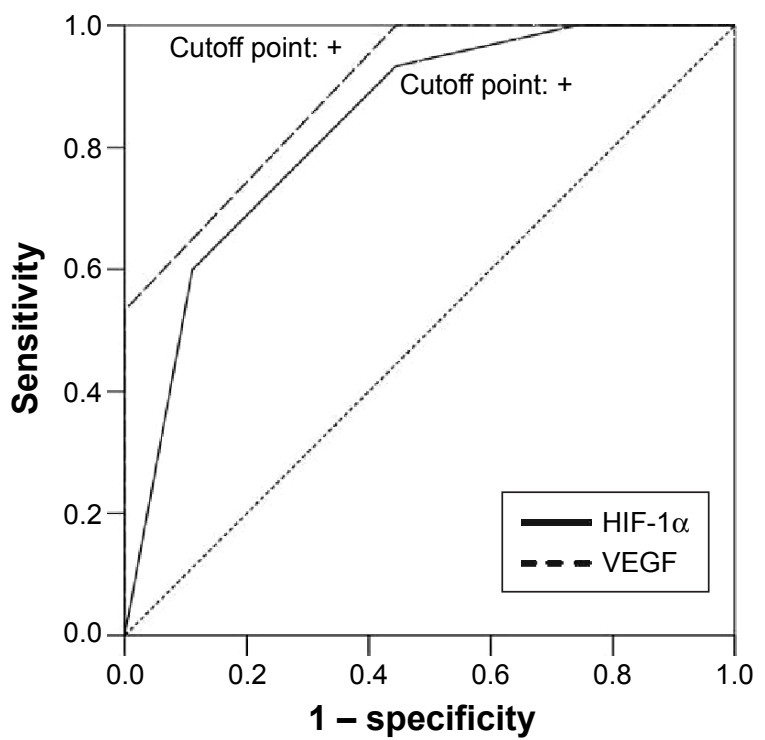

Figure 3 ROC curves for VEGF and HIF-I $\alpha$ expression for predicting PLNM in patients with LACC before PRCT.

Abbreviations: HIF-I $\alpha$, hypoxia inducible factor-I $\alpha$; LACC, locally advanced cervical cancer; PLNM, pelvic lymph node metastasis; PRCT, preoperative chemoradiotherapy; ROC, receiver-operating characteristic; VEGF, vascular endothelial growth factor. value was $76.92 \%$, and the negative predictive value was $82.76 \%$. The diagnostic accuracy was $80.95 \%$.

\section{Discussion}

In recent years, neoadjuvant chemotherapy and concomitant PRCT have been used for the treatment of LACC. Concomitant PRCT is not a simple combination of radiotherapy and chemotherapy. The aim of PRCT is to reduce tumor lesions and improve parametrial conditions, thereby reducing the clinical stage. This strategy is more favorable for complete tumor resection, results in a better negative surgical margin, and reduces intraoperative dissemination and postoperative metastasis. In addition, this procedure eliminates micrometastases and subclinical lesions. PCRT is especially suited for young patients with cancer who want to preserve ovarian and vaginal function. ${ }^{8,9}$ However, varying PRCT responses have been documented, and the findings are often conflicting. ${ }^{2,3}$

In addition, although PRCT might offer a valuable opportunity for subsequent radical surgery, the nonresponsive rate and the adverse effects of PRCT should also be considered by surgeons before making the treatment plan. Therefore, biomarkers for predicting PRCT sensitivity and prognosis in patients with LACC are of high importance. Unfortunately, to date, little effort has been made toward the identification of biomarkers. The availability of these biomarkers would allow for the identification of patients who would benefit from PRCT and allow surgeons to select appropriate patients and provide individualized treatment strategies.

In the current study, we first observed the clinical effect of PRCT among 43 patients with LACC and then further investigated the prognostic significance of VEGF and HIF- $1 \alpha$ for predicting PRCT responses and positive findings of PLNM, which might be a poor prognostic factor for patients with cervical cancer. Among the patients, 42 of 43 had the option of surgery after PRCT, and five patients achieved a $\mathrm{CR}$, where the cervix and vaginal vault recovered with normal morphology. The postoperative pathological examination of these five patients further revealed the absence of any tumor lesions. Moreover, 30 patients had a PR, resulting in a total response rate of $81.39 \%$ (35/43). In a study of 174 patients with LACC, ${ }^{2} 121$ patients received PRCT, and the other 53 received preoperative radiotherapy alone. In their study, the PRCT group achieved a higher pathological CR rate $(31.4 \%)$ than the simple radiotherapy group $(28.3 \%$; $P=0.724$ ), and the $\mathrm{CR}$ rate was even higher when the tumor volume was $<5 \mathrm{~cm}(38.2 \%$ vs $30.8 \% ; P=0.623)$. Our study is consistent with the study by Wei et al. ${ }^{2}$ Concomitant 
PRCT can significantly improve the surgical rate and plays a positive role by providing the option for radical surgery in young patients. ${ }^{2}$

In our study, we also evaluated the expression of VEGF and HIF- $1 \alpha$ before and after PRCT. VEGF is currently known as a key pro-angiogenic factor that is often overexpressed in malignant tissue. The VEGF expression level reflects the ability of tumor angiogenesis, growth, invasion, and metastasis and is closely associated with lymph node metastasis in cervical cancer. ${ }^{10}$ Chen et al $^{11}$ reported that a reduction in VEGF expression in cervical cancer cells could inhibit migration, invasion, and adhesion ability while promoting apoptosis. These processes are favorable for stabilizing the disease and improving the prognosis of patients with cervical cancer. Along with VEGF, HIF-1 $\alpha$ is an important transcription factor and also plays a key role in regulating neovascularization. HIF- $1 \alpha$ is always upregulated to maintain stable oxidative metabolism and promote the growth and metastasis of cancer cells under hypoxic conditions. ${ }^{12}$ The interaction between VEGF and HIF- $1 \alpha$ may play a critical role in the development, local invasion, and distant metastasis of cervical cancer. ${ }^{4}$

VEGF and HIF- $1 \alpha$ expression before PRCT was significantly higher than after PRCT (VEGF: $85.71 \%$ vs $66.67 \%$; HIF-1 $\alpha$ : $83.33 \%$ vs $59.52 \% ; P<0.05)$. In CR patients, the expression of VEGF and HIF- $1 \alpha$ was negative. In PR patients, their expression levels were $70 \%$ and $63.33 \%$, respectively, after PRCT. It was obvious in our study that treatment with PRCT significantly reduced the expression of VEGF and HIF- $1 \alpha$ in patients with LACC. Considering the association between the expression of these markers and the clinical response to PRCT, the expression of VEGF and HIF- $1 \alpha$ can reflect the progression of LACC and provide reference indices for evaluating LACC malignancy. Similar to our study, Chen et al evaluated the predictive value of VEGF and HIF-1 $\alpha$ to neoadjuvant chemotherapy response in adolescent osteosarcoma. The authors found that HIF-1 $\alpha$ has a sensitivity of $64.7 \%$ and a specificity of $71.9 \%$ for predicting neoadjuvant chemotherapy response, thus showing a close association with VEGF. ${ }^{13}$

Although the FIGO and NCCN guidelines still adopt preoperative clinical staging for cervical cancer, PLNM is generally considered a postoperative high-risk factor for patients with cervical cancer, which affects their prognosis and requires adjuvant RCT after surgery. PLNM is indeed important in patients with LACC. LACC lesions are relatively large and can easily cause local invasion involving lymph nodes. If the presence of lymph node metastasis can be accurately predicted before PRCT, it is possible to prevent radical RCT following radical surgery and change to radical chemotherapy instead in time, which may promptly reduce the suffering of patients while avoiding RCT and surgical complications. In our study, the VEGF and HIF-1 $\alpha$ expression rates in the PLNM group were significantly higher than those of the non-PLNM group $(P<0.05)$. Thus, VEGF and HIF-1 $\alpha$ overexpression may be associated with PLNM in patients with LACC, thereby further affecting the prognosis of patients. Hutchison et al suggested that HIF-1 $\alpha$ was mainly expressed in cervical cancer tissue and that higher HIF-1 $\alpha$ expression in larger tumors was closely associated with patient prognosis. ${ }^{14} \mathrm{Du}$ et al observed that tumor size and serum VEGF levels are both independent risk factors for treatment prognosis in patients with cervical cancer with tumor sizes $>4 \mathrm{~cm} .{ }^{15}$ Moreover, in our study, the diagnosis of PLNM by pre-PRCT VEGF and HIF- $1 \alpha$ protein expression scores yielded an area under the curve of 0.896 and 0.835 , respectively. Using a combination of VEGF and HIF- $1 \alpha$ expression could achieve a relatively high sensitivity of $66.67 \%$ and specificity of $88.89 \%$. VEGF and HIF- $1 \alpha$ are closely associated with the development, progression, and prognosis of cervical cancer. Thus, VEGF and HIF-1 $\alpha$ may serve as potential biomarkers for predicting the prognosis of LACC.

This scenario suggests that VEGF and HIF-1 $\alpha$ may be therapeutic targets for adjuvant LACC therapy. As a biomarker, VEGF-targeted therapy has been previously investigated, and bevacizumab in combination with chemotherapy can alleviate the recurrence and extend the overall survival period in patients with metastatic cervical cancer. ${ }^{15}$ However, the effect of VEGF-targeted therapies on the specific subtype of LACC is still unclear. Furthermore, the intrinsic biological association between HIF-1 $\alpha$ and PLNM remains to be elucidated. Therefore, more research is required to comprehensively evaluate the possibility of lymph node metastasis in patients with high expression levels of VEGF and HIF-1 $\alpha$ before PRCT to fully assess the clinical stage of these patients and make treatment strategies.

Although this was a retrospective study with a limited sample size and follow-up duration, we were able to identify potential markers for predicting the PRCT response and prognosis in patients with LACC. The results of the current study are of potential clinical value and indicate that serial detection of VEGF and HIF-1 $\alpha$ expression has a high sensitivity and specificity for predicting tumor recurrence and prognosis in patients with LACC. Future studies with larger sample sizes and long-term follow-ups are warranted to confirm our findings. There is still ample room for improvement in the sensitivity and specificity. 


\section{Conclusion}

We have demonstrated that PRCT could achieve good clinical response in patients with LACC. In patients with LACC, VEGF and HIF- $1 \alpha$ expression before PRCT was significantly higher than after PRCT and the change in VEGF and HIF- $1 \alpha$ expression for the same individual pre and post PRCT was linked to their clinical responses. In addition, serial detection of VEGF and HIF- $1 \alpha$ demonstrated a sensitivity of $66.67 \%$ and specificity of $88.89 \%$ in diagnosing PLNM in patients with LACC. These findings suggest that VEGF and HIF-1 $\alpha$ are potential biomarkers for PRCT and have great clinical significance for the prediction of PRCT response and prognosis in patients with LACC.

\section{Acknowledgments}

This project was supported partially by Science and Technology Development Fund of Nanjing Medical University (2014NJ-MU021 to Zhu PF), Scientific Research Project from the Association of Maternal and Child Health of Jiangsu province (FYX201507 to Zhu PF), and Shanghai Natural Science Fund (13ZR1451900 to Yuan L).

The abstract of this paper was presented at the fifteenth academic conference of gynecology and obstetrics in Jiangsu province as a poster presentation with interim findings.

\section{Disclosure}

The authors report no conflicts of interest in this work.

\section{References}

1. Martinelli F, Bogani G, Ditto A, et al. How often parametrial involvement leads to post-operative adjuvant treatment in locally advanced cervical cancer after neoadjuvant chemotherapy and type $\mathrm{C}$ radical hysterectomy? Eur J Surg Oncol. 2015;41(8):1089-1096.

2. Wei L-C, Wang N, Shi M, et al. Clinical outcome observation of preoperative concurrent chemoradiotherapy/radiotherapy alone in 174 Chinese patients with local advanced cervical carcinoma. Onco Targets Ther. $2013 ; 6: 67-74$
3. Ushijima K, Fujiyoshi K, Kawano K, et al. Concurrent chemoradiotherapy with low-dose daily cisplatin for high risk uterine cervical cancer: a long-term follow-up study. J Gynecol Oncol. 2013;24(2): 108-113.

4. Kim NS, Kang Y-J, Jo J-O, et al. Elevated expression of thymosin $\beta 4$, vascular endothelial growth factor (VEGF), and hypoxia inducible factor (HIF)-1 $\alpha$ in early-stage cervical cancers. Pathol Oncol Res. 2011; 17(3):493-502.

5. Cheng W, Zhou W. Clinical significance and relationship between HIF-1alpha, COX-2, VEGF and microvessel density expression in laryngeal carcinoma. Lin Chung Er Bi Yan Hou Tou Jing Wai Ke Za Zhi. 2010;24(18):820-822.

6. Kang S, Nam BH, Park JY, et al. Risk assessment tool for distant recurrence after platinum-based concurrent chemoradiation in patients with locally advanced cervical cancer: a Korean gynecologic oncology group study. J Clin Oncol. 2012;30(19):2369-2374.

7. Qiu Y, Zhou H. Expression of HIF-1alpha and VEGF in human laryngeal carcinoma and its relationship with angiogenes. Lin Chung Er Bi Yan Hou Tou Jing Wai Ke Za Zhi. 2014;28(6):389-393.

8. Bazaeva I, Gorbunova V, Kravets O, et al. Chemoradiotherapy for locally advanced cervical cancer. Vopr Onkol. 2013;60(3):280-287.

9. Yuan G, Wu L, Huang M, Li N, An J. A phase II study of concurrent chemo-radiotherapy with weekly nedaplatin in advanced squamous cell carcinoma of the uterine cervix. Radiat Oncol. 2014;9(1):55.

10. Vici P, Mariani L, Pizzuti L, et al. Emerging biological treatments for uterine cervical carcinoma. J Cancer. 2014;5(2):86-97.

11. Chen L, Wu YY, Liu P, et al. Down-regulation of HPV18 E6, E7, or VEGF expression attenuates malignant biological behavior of human cervical cancer cells. Med Oncol. 2011;28(suppl 1):S528-S539.

12. Fu SL, Miao J, Ding B, et al. A polymorphism in the $3^{\prime}$ untranslated region of hypoxia-inducible factor-1 alpha confers an increased risk of cervical cancer in a Chinese population. Neoplasma. 2014;61(1): 63-69.

13. Chen Y, Yang Y, Yuan Z, Wang C, Shi Y. Predicting chemosensitivity in osteosarcoma prior to chemotherapy: an investigational study of biomarkers with immunohistochemistry. Oncol Lett. 2012;3(5): 1011-1016.

14. Hutchison GJ, Valentine HR, Loncaster JA, et al. Hypoxia-inducible factor 1alpha expression as an intrinsic marker of hypoxia: correlation with tumor oxygen, pimonidazole measurements, and outcome in locally advanced carcinoma of the cervix. Clin Cancer Res. 2004;10(24): 8405-8412.

15. Du K, Gong HY, Gong ZM. Influence of serum VEGF levels on therapeutic outcome and diagnosis/prognostic value in patients with cervical cancer. Asian Pac J Cancer Prev. 2014;15(20):8793-8796.
OncoTargets and Therapy

\section{Publish your work in this journal}

OncoTargets and Therapy is an international, peer-reviewed, open access journal focusing on the pathological basis of all cancers, potential targets for therapy and treatment protocols employed to improve the management of cancer patients. The journal also focuses on the impact of management programs and new therapeutic agents and protocols on

\section{Dovepress}

patient perspectives such as quality of life, adherence and satisfaction. The manuscript management system is completely online and includes a very quick and fair peer-review system, which is all easy to use. Visit http://www.dovepress.com/testimonials.php to read real quotes from published authors. 